\title{
Information Theory-Based Shot Cut/Fade Detection and Video Summarization
}

\author{
Zuzana Černeková, Ioannis Pitas, Senior Member, IEEE, and Christophoros Nikou, Member, IEEE
}

\begin{abstract}
New methods for detecting shot boundaries in video sequences and for extracting key frames using metrics based on information theory are proposed. The method for shot boundary detection relies on the mutual information (MI) and the joint entropy (JE) between the frames. It can detect cuts, fade-ins and fade-outs. The detection technique was tested on the TRECVID2003 video test set having different types of shots and containing significant object and camera motion inside the shots. It is demonstrated that the method detects both fades and abrupt cuts with high accuracy. The information theory measure provides us with better results because it exploits the inter-frame information in a more compact way than frame subtraction. It was also successfully compared to other methods published in literature. The method for key frame extraction uses MI as well. We show that it captures satisfactorily the visual content of the shot.
\end{abstract}

Index Terms-Detection accuracy, entropy, key frame extraction, mutual information (MI), shot boundary detection, video analysis, video segmentation.

\section{INTRODUCTION}

$\mathbf{I}$ NDEXING and retrieval of digital video is an active research area. Video segmentation is a fundamental step in analyzing video sequence content and in devising methods for efficient access, retrieval and browsing of large video databases. Shot boundary detection is an important task in managing video databases for indexing, browsing, search, summarization and other content-based operations. A video shot is defined as a sequence of frames captured by one camera in a single continuous action in time and space [1]. Usually it is a group of frames that have consistent visual characteristics (including color, texture and motion). Video shots are the basic structural building blocks of a video sequence. Their boundaries need to be determined possibly automatically to allow content-based video manipulation.

Early work on shot detection was mainly focused on abrupt cuts. A comparison of existing methods is presented in [2] and [3]. In some early approaches a cut is detected when a certain difference measure between consecutive frames exceeds a

Manuscript received February 12, 2004; revised November 3, 2004. This work has been supported by the Commission of the European Communities in the framework of the Methods for Unified Multimedia Information Retrieval (MOUMIR) Project RTN-1999-00177 MOUMIR. The C-SPAN video used in this work is provided for research purposes by C-SPAN through the TREC Information-Retrieval Research Collection. C-SPAN video is copyrighted. This paper was recommended by Associate Editor R. Lienhart.

Z. Černeková and I. Pitas are with the Aristotle University of Thessaloniki, Department of Informatics, Artificial Intelligence and Information Analysis Laboratory, 54006 Thessaloniki, (e-mail: zuzana@aiia.csd.auth.gr; pitas@aiia.csd.auth.gr).

C. Nikou is with the University of Ioannina, Department of Computer Science, GR 45110 Ioannina, Greece (e-mail: cnikou@cs.uoi.gr).

Digital Object Identifier 10.1109/TCSVT.2005.856896 threshold. The difference measure is computed either at a pixel level or at a block level. Noticing the weakness of pixel difference methods (high sensitivity to object and camera motions), many researchers suggested the use of other measures based on global information, such as intensity histograms or color histograms [4]-[7]. The standard color histogram-based algorithm and its variations are widely used for detecting abrupt cuts. Even these histograms do not explicitly model the image difference caused by large camera motion, and thus, strictly speaking, are incapable to differentiate between smooth camera motion/parameter changes and gradual scene transitions. While the use of more complex features, such as image edges or histograms or motion vectors [8] improves the situation, it will only relieve but do not solve this problem [9]. The possibility of detecting abrupt cuts by measuring information changes between adjacent images, quantized by mutual information (MI) in gray-scale space of the images is followed in [10]. In order to detect the video shot cut they are using affine image registration for compensation of camera panning and zooming. This makes the approach very computationally expensive.

Gradual transitions such as dissolves, fade-ins, fade-outs and wipes are examined in [11]-[17]. Such transitions are generally more difficult to detect, due to camera and/or object motions within a shot. A fade is a transition of gradual decrease (fade-out) or increase (fade-in) of visual intensity. Fades are widely used in TV footage and their appearance generally signals a shot or story change. Therefore, their detection is a very powerful tool for shot classification and story summarization. Existing techniques for fade detection proposed in the literature rely on twin thresholding [18] or standard deviation of pixel intensities [2]. Hampapur et al. [19] suggested a shot detection scheme based on modeling video edits. They computed a chromatic images by dividing the intensity change of each pixel between two successive frames by the intensity of the same pixel in the second frame. During dissolves and fades, this chromatic image assumes a reasonably constant value. This technique is very sensitive to camera and object motion. Lienhart [2] proposed to locate first all monochromatic frames in the video as potential start/end points of fades. Monochromatic frames were identified as frames with standard deviation of pixel intensities close to zero. Fades were then detected by starting to search in both directions for a linear increase in the standard deviation of pixel intensity/color. An average true positive rate of $87 \%$ was reported at a false alarm rate of $30 \%$. An alternative approach also based on the variance of pixel intensities was proposed by Alattar [20]. Fades were detected first by recording all negative spikes in the time series of the second order difference of the pixel intensity variance, and then by ensuring that the first 
order difference of the mean of the video sequence was relatively constant next to the above mentioned negative spike. A combination of both approaches is described in Truong et al. [21]. These methods have a relatively high false detection rate. Moreover, even if the existing methods based on histograms [22] detect scene changes correctly, they cannot detect fades and other gradual video transitions.

Key frames provide a suitable video summarization and a framework for video indexing, browsing and retrieval. The use of key frames greatly reduces the amount of data required in video indexing and provides an organizational framework for dealing with the video content. A lot of research work has been done in key frame extraction [23]-[25]. The simplest proposed methods are choosing only one frame for each shot (usually the first one), regardless of the complexity of visual content. The more sophisticated approaches take into account visual content, motion analysis and shot activity [26]. These approaches either do not effectively capture the major visual content or are computationally expensive.

In this paper, we propose a new approach for shot boundary detection in the uncompressed image domain based on the MI and the joint entropy (JE) between consecutive video frames. Mutual information is a measure of information transported from one frame to another one. It is used for detecting abrupt cuts, where the image intensity or color is abruptly changed. A large video content difference between two frames, showing weak inter-frame dependency leads to a low MI. The entropy measure provides us with better results, because it exploits the inter-frame information flow in a more compact way than a frame subtraction. In the case of a fade-out, where the visual intensity is usually decreasing to a black image, the decreasing inter-frame JE is used for detection. In case of a fade-in, the increasing JE is used for detection. The application of these entropy-based techniques for shot cut detection was experimentally proven to be very efficient, since they produce false acceptance rates very close to zero.

The proposed method was also favorably compared to other recently proposed shot cut detection techniques. At first, we compared the JE metric for fade detection to the technique proposed by Lienhart [2] relying on the standard deviation of pixel intensities. Finally, we compared our algorithm for abrupt cut detection based on MI to two techniques relying on histograms. The first one [22] combines two shot boundary detection schemes based on color frame differences and color vector histogram differences between successive frames. The second technique [2] uses one of the most reliable variants of histogram-based detection algorithms.

We propose also a method for extracting key frames from each shot using already calculated MI values. The MI expresses the changes in the shot and thus, the selected key frames capture well the visual content of the shot.

The remainder of the paper is organized as follows. In Section II, a brief description of the MI and the JE as well as a definition of abrupt cuts and fades are presented. The description of our approach and its computational complexity are addressed in Section III. In Section IV, the method for key frame extraction is described. Experimental results are presented and commented in Section $\mathrm{V}$ and conclusions are drawn in Section VI.

\section{BACKGROUND AND DEFINITIONS}

\section{A. Mutual Information}

Let $X$ be a discrete random variable with a set of possible outcomes $A_{X}=\left\{a_{1}, a_{2}, \ldots a_{N}\right\}$ having probabilities $\left\{p_{1}, p_{2}, \ldots p_{N}\right\}$, with $p_{X}\left(x=a_{i}\right)=p_{i}, p_{i} \geq 0$ and $\sum_{x \epsilon A_{X}} p_{X}(x)=1$. Entropy measures the information content or "uncertainty" of $X$ and it is given by [27] and [28]

$$
H(X)=-\sum_{x \in A_{X}} p_{X}(x) \log p_{X}(x)
$$

The JE of $X, Y$ is expressed as

$$
H(X, Y)=-\sum_{x, y \in A_{X}, A_{Y}} p_{X Y}(x, y) \log p_{X Y}(x, y)
$$

where $p_{X Y}(x, y)$ is the joint probability density function. For two random variables $X$ and $Y$, the conditional entropy of $Y$ given $X$ is written $H(Y \mid X)$ and is defined as

$$
\begin{aligned}
H(Y \mid X) & =\sum_{x \in A_{X}} p_{X}(x) H(Y \mid X=x)= \\
& =-\sum_{x, y \in A_{X}, A_{Y}} p_{X Y}(x, y) \log p_{X Y}(x \mid y)
\end{aligned}
$$

where $p_{X Y}(x \mid y)$ denotes conditional probability. The conditional entropy $H(Y \mid X)$ is the uncertainty in $Y$ given knowledge of $X$. It specifies the amount of information that is gained by measuring a variable and already knowing another one. It is very useful if we want to know if there is a functional relationship between two data sets. Conditional entropy has the following properties:

- $H(X \mid Y) \leq H(X)$;

- $H(X \mid Y)=H(Y \mid X)$;

- $H(X \mid Y)=0 \Leftrightarrow X=f(Y)$.

The MI between the random variables $X$ and $Y$ is given by

$$
I(X, Y)=-\sum_{x, y \in A_{X}, A_{Y}} p_{X Y}(x, y) \log \frac{p_{X Y}(x, y)}{p_{X}(x) p_{Y}(y)}
$$

and measures the amount of information conveyed by $X$ about $Y$. Some important properties of the MI are as follows.

- $I(X, Y) \geq 0$.

- For both independent and zero entropy sources $X$ and $Y$ : $I(X, Y)=0$.

- $I(X, Y)=I(Y, X)$

- The relation between the MI and the JE of random variables $X$ and $Y$ is given by

$$
I(X, Y)=H(X)+H(Y)-H(X, Y)
$$

where $H(X)$ and $H(Y)$ are the marginal entropies of $X$ and $Y$.

- The MI is a measure of the additional information known about one expression pattern when given another as is given by

$$
I(X, Y)=H(X)-H(X \mid Y) .
$$

According to (5), the MI not only provides us with a measure of correspondence between $X$ and $Y$ but also takes into account the information carried by each frame at their overlap. By these means, MI decreases when the amount of shared information 


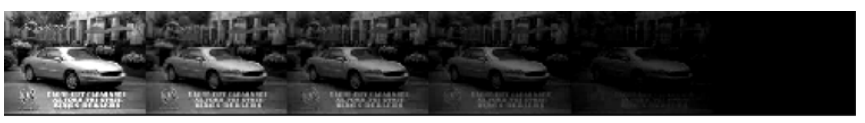

(a)

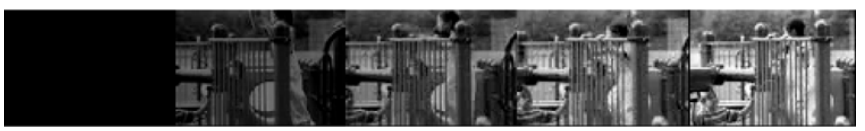

(b)

Fig. 1. Consecutive frames from "news" video sequence showing: (a) fade-out and (b) fade-in.

between $H(X)$ and $H(Y)$ is small. We can also see from (6) that the MI will reduce if $X$ carries no information about $Y$.

\section{B. Video Cuts and Fades}

A video shot cut (abrupt cut) is an instantaneous content transition from one shot to the next one. It is obtained by simply concatenating two different shots without the use of any other transition effect. The cut boundaries show an abrupt change in image intensity or color. Cuts between shots with little content or camera motion and constant illumination conditions can be easily detected by looking for sharp intensity changes. However, in the presence of fast object motion, camera motion or illumination changes, it is difficult to distinguish if intensity changes are due to shot content changes or a shot cut [18].

A fade-out is a video transition determining the progressive darkening of a shot until the last frame becomes black, as can be seen in Fig. 1(a). A fade-in allows the gradual transition from a black frame to the fully illuminated one, as shown in Fig. 1(b). Fades spread the boundary between two shots across a number of consecutive video frames. They have both start and end frames identifying the transition sequence. In both cases (fade-in, fade-out) fades can be mathematically modeled as luminance scaling operations. If $G(x, y, t)$ is a gray scale sequence and $l_{s}$ is the length of the transition sequence, an intensity scaling of $G(x, y, t)$ is modeled as [18]

$$
f(x, y, t)=G(x, y, t) \cdot\left(1-\frac{t}{l_{s}}\right), \quad t \in\left[t_{0}, t_{0}+l_{s}\right] .
$$

Therefore, fade-out is modeled by

$$
f(x, y, t)=G_{1}(x, y, t) \cdot\left(\frac{l_{1}-t}{l_{1}}\right)
$$

and fade-in by

$$
f(x, y, t)=G_{2}(x, y, t) \cdot\left(\frac{t}{l_{2}}\right) .
$$

\section{ShOt DeteCtion}

In our approach, the MI and the JE between two successive frames are calculated separately for each of the RGB components. Let us consider that the video sequence gray levels vary from 0 to $N-1$. At frame $\mathbf{f}_{t}$ three $N \times N$ matrices $\mathbf{C}_{t, t+1}^{R}$, $\mathbf{C}_{t, t+1}^{G}$ and $\mathbf{C}_{t, t+1}^{B}$ are created carrying information on the gray level transitions between frames $\mathbf{f}_{t}$ and $\mathbf{f}_{t+1}$. In the case of the $R$ component, the element $\mathbf{C}_{t, t+1}^{R}(i, j)$, with $0 \leq i \leq N-1$ and

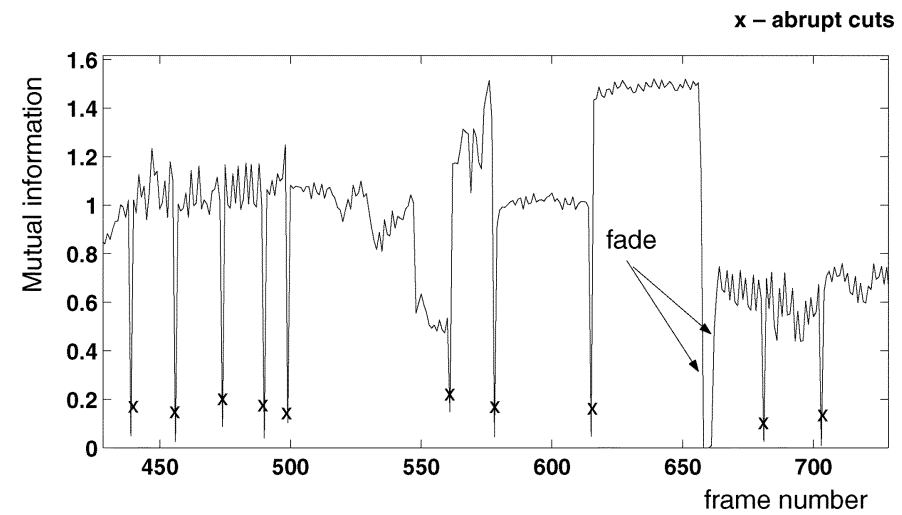

Fig. 2. Time series of the MI from "ABC news" video sequence showing abrupt cuts and one fade.

$0 \leq j \leq N-1$, corresponds to the probability that a pixel with gray level $i$ in frame $\mathbf{f}_{t}$ has gray level $j$ in frame $\mathbf{f}_{t+1}$. By the other words, $\mathbf{C}_{t, t+1}^{R}(i, j)$ is a number of pixels which change from gray level $i$ in frame $\mathbf{f}_{t}$ to gray level $j$ in frame $\mathbf{f}_{t+1}$, divided by the number of pixels in the video frame. Following (4), the MI $I_{t, t+1}^{R}$ of the transition from frame $\mathbf{f}_{t}$ to frame $\mathbf{f}_{t+1}$ for the $R$ component is expressed by

$$
I_{t, t+1}^{R}=-\sum_{i=0}^{N-1} \sum_{j=0}^{N-1} \mathbf{C}_{t, t+1}^{R}(i, j) \log \frac{\mathbf{C}_{t, t+1}^{R}(i, j)}{\mathbf{C}_{t}^{R}(i) \mathbf{C}_{t+1}^{R}(j)} .
$$

The total MI is defined as

$$
I_{t, t+1} \triangleq I_{t, t+1}^{R}+I_{t, t+1}^{G}+I_{t, t+1}^{B} .
$$

By using the same considerations, the JE $H_{t, t+1}^{R}$ of the transition from frame $\mathbf{f}_{t}$ to frame $\mathbf{f}_{t+1}$, for the $R$ component, is given by

$$
H_{t, t+1}^{R}=-\sum_{i=0}^{N-1} \sum_{j=0}^{N-1} \mathbf{C}_{t, t+1}^{R}(i, j) \log \mathbf{C}_{t, t+1}^{R}(i, j) .
$$

The total JE is defined as

$$
H_{t, t+1} \triangleq H_{t, t+1}^{R}+H_{t, t+1}^{G}+H_{t, t+1}^{B} .
$$

\section{A. Abrupt Shot Cut Detection}

A small value of the MI $I_{t, t+1}$ indicates the existence of a cut between frames $f_{t}$ and $f_{t+1}$. Basically, in this context, abrupt cut detection is the outlier detection in an one-dimensional MI signal given by (11) [29]. In order to detect possible shot cuts, an adaptive thresholding approach was employed. Local MI mean values on an one-dimensional temporal window $W$ of size $N_{W}$ are obtained at each time instance $t_{c}$ without considering the current value $I_{t_{c}, t_{c}+1}$ at the current window center $t_{c}$ [29]

$$
\bar{I}_{t_{c}}=\frac{1}{N_{W}} \sum_{\substack{t \in W \\ t \neq t_{c}}} I_{t, t+1}
$$

The quantity $\bar{I}_{t_{c}} / I_{t_{c}, t_{c}+1}$ is then compared to a threshold $\epsilon_{c}$ in order to detect the peaks, which correspond to the shot cuts. Threshold $\epsilon_{c}$ was chosen experimentally. An example of abrupt cut detection using MI is illustrated in Fig. 2. 


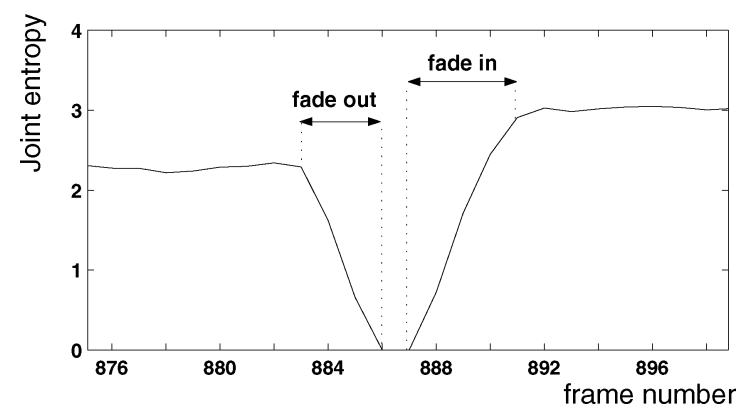

Fig. 3. JE signal from "CNN news" video sequence showing a fade-out and fade-in to the next shot.

\section{B. Fade Detection}

Since MI decreases when the transmitted information from one frame to another is small (in case of cuts and fades) the JE (13) is employed, to efficiently distinguish fades from cuts. The JE measures the amount of information carried by the union of these frames. Therefore, its value decreases only during fades, where a weak amount of inter-frame information is present. The pattern showing fade out and fade in is shown on Fig. 3. Thus, only the values of $H_{t, t+1}$ below a certain threshold $\epsilon_{f}$ are examined. These values correspond to the black frames. The instance, where the JE is at a local minimum, is detected and is characterized as the end time instance $t_{e}$ of the fade-out. At this point the frame has become black, it does not carry any information. The next step consists in searching for the fade-out start point $t_{s}$ in the previous frames using the criterion

$$
\frac{H_{t_{s}, t_{s}+1}-H_{t_{s}-1, t_{s}}}{H_{t_{s}-1, t_{s}}-H_{t_{s}-2, t_{s}-1}} \geq T
$$

where $T$ is a predefined threshold which guarantees that, at start point $t_{s}$, the JE starts decreasing. In order to handle a specific type of video sequences where the frame content remains exactly the same for two or three consecutive frames, due to the chosen video digitalization procedure (typically a reduced digitization frame rate), we check the possible increase of JE values up to the third frame. The same procedure also applies for fade-in detection (with $t_{s}$ being detected at first). Finally, since the fade has boundary spread across number of frames, the segment is considered as a fade only if $t_{e}-t_{s} \geq 2$, otherwise it is labeled as a cut. An example of JE signal showing a fade-out detection is presented in Fig. 4.

\section{Computational Complexity}

The computational complexity of these algorithms consist of calculating three histograms for each color component $R, G, B$ for two consecutive frames. If the frame size is $n$ pixels than we need $n$ additions to calculate one histogram. First we need to calculate three histograms, which consist of $3 n$ additions. We also need another $3 N^{2}$ multiplications, $(N-1)^{2}$ additions and $N^{2}$ logarithm calculations for computing (10), where $N$ is number of video sequence gray levels separately for $R, G$, and $B$ color channels. Thus, for calculation the MI between two frames $O\left(n+N^{2}\right)$ additions, $O\left(N^{2}\right)$ multiplications and $O\left(N^{2}\right) \log$ arithm calculations are needed. The same order of computational complexity is needed for the calculation of JE, namely

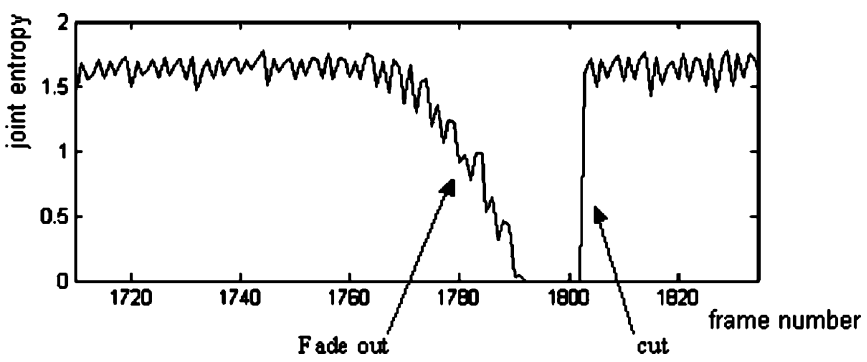

Fig. 4. JE signal from "basketball" video sequence showing a fade-out and a transition from a black frame to the next shot.

$3\left(n+(N-1)^{2}\right)+2$ additions, $3 N^{2}$ multiplications and $3 N^{2}$ logarithm calculations.

\section{Key Frame SELECTION}

After the temporal segmentation of a video sequence to shots, the key frames can be selected from each shot for video indexing. Our approach uses MI values, which provided us information about content changes between consecutive frames in the shot. Let us have a video shot having $N_{L}$ frames $s=$ $\left\{f_{1}, f_{2}, \ldots, f_{N_{L}}\right\}$ obtained by our method for shot cut detection [30] described in the Section III. Let the MI values in this shot be $I_{s}=\left\{I_{1,2}, I_{2,3}, \ldots, I_{N_{L}-1, N_{L}}\right\}$. In order to find if the content in the shot changes significantly, the standard deviation $\sigma_{I_{s}}$ of the MI within this shot is calculated. The value $\sigma_{I_{s}}$ is compared to predefined threshold $\epsilon$. If $\sigma_{I_{s}}<\epsilon$, we assume that the content did not change significantly during the shot. Thus, any frame can effectively represent the visual content. Such a shot can present for example, a anchor person in a news video sequence. In this case, the first or a middle frame is selected as key frame.

In the case of bigger content changes within the shot, the shot must be described by more than one key frame. This is done by a split-merge approach. The MI values in the shot are divided into clusters $\left\{c_{i}\right\}_{i=1}^{K}$, where $K$ is a number of clusters obtained after the split-merge algorithm. The threshold parameter $\delta$ controls the number of created frame clusters. Initially, all the MI values in the shot are assigned to the one cluster $c_{1}=$ $\left\{I_{1,2}, I_{2,3}, \ldots, I_{N_{L}-1, N_{L}}\right\}$. The standard deviation $\sigma_{c_{1}}$ of these values in the cluster is compared to the predefined threshold $\delta$. If it exceeds the threshold, $\sigma_{c_{1}}>\delta$, all the MI values are split in two clusters: $c_{11}=\left\{I_{1,2}, I_{2,3}, \ldots, I_{N_{L} / 2-1, N_{L} / 2}\right\}$ and $c_{12}=\left\{I_{N_{L} / 2, N_{L} / 2+1}, I_{N_{L} / 2+1, N_{L} / 2+2}, \ldots, I_{N_{L}-1, N_{L}}\right\}$. The algorithm works recursively till the standard deviation of the MI values in the cluster is smaller than the given threshold $\delta$. Then, consecutive clusters are checked for possible merging. If the standard deviation of two consecutive clusters is smaller than threshold $\delta$, these clusters are merged. This way, all frames from the given shot are split to clusters, depending on the MI values. After this procedure, only those clusters having enough frames are considered as key clusters [26] and a representative frame is extracted from this cluster as the potential key frame. In this paper, key cluster should have more than $N_{L} /(K * 2)$ frames, where $N$ is number of frames in the shot and $K$ is number of clusters. In [26], a key cluster should have at least $N_{L} / K$ frames. However, in the case when we have only two clusters, 


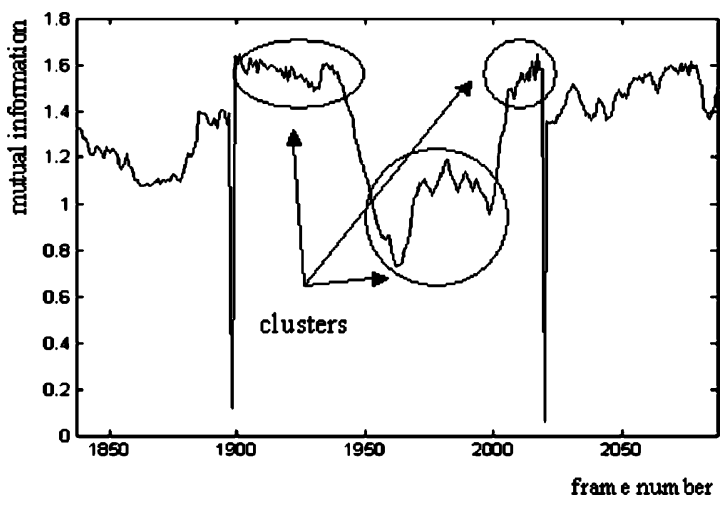

Fig. 5. MI signal from "star" video sequence presenting the clusters created by split-merge method. The selected potential key frames from each cluster is shown in Fig. 6.

this method will discard the smaller one, which is not acceptable. For each key cluster the most representative frame is taken as a potential key frame. The most representative frame is the one which maximizes inter-frame MI in the cluster

$$
f_{\text {key }}=\arg \max _{\bar{f}}\left(\frac{1}{N_{j}} \sum_{\substack{i \\ \bar{f} \neq f_{i}}}^{N_{j}} I_{\bar{f}, f_{i}}\right)
$$

where $N_{j}$ is number of frames in the cluster, we call this frame median frame. An example of this procedure can be seen in Fig. 5.

After extracting potential key frames $\left\{k_{i}\right\}_{i=1}^{K}$ from the shot $s$, we try to reduce the number of key frames that represent the shot. To do this, these key frames are compared to each other by calculating their MI using (11). If the content of the key frames is similar enough (as indicated by a high MI value), the shot can be presented by less key frames. Therefore, if $I_{k_{i}, k_{i+1}}>\epsilon$, where $\epsilon$ is a predefined threshold, only the frame $k_{i+1}$ is considered to be a key frame and is compared to the next potential key frame $k_{i+2}$. Otherwise, both frames $k_{i}$ and $k_{i+1}$ are taken as key frames and $k_{i+1}$ is further compared to the others potential key frames $\left\{k_{i+2}, \ldots, k_{K}\right\}$. An example can be seen in Fig. 6. After calculating the MI between these frames, only the first frame (frame number 1904) was selected as a key frame to represent content of the shot. This procedure of the reduction of key frame number enhances the robustness of our method to the choice of threshold $\delta$ used in the split-merge procedure. Let us note that we are not interested only in picking the fewest possible key frames but also in picking good key frames that are visually and possibly semantically important.

The reduction of the number of key frames can be done by using Median LVQ [31]. This way, we can handle the problem when the potential key frames are similar in the beginning and in the end of the shot. At first we assign all the potential key frames to one cluster $c_{1}$. Then the key frame that maximizes inter-frame $\mathrm{MI}$ is chosen as representative key frame $k_{1}$ of this cluster. We find the key frame $k$, which minimizes the MI between the representative key frame $k_{1}$ and other key frames. If this MI is below a given threshold, we split the cluster to the two. The key frame $k$, is chosen as a representative $k_{2}$ for new

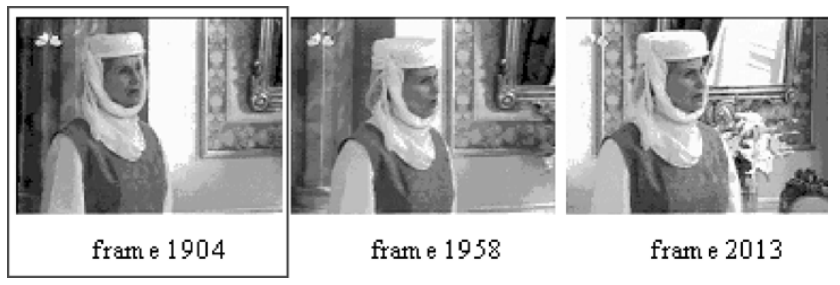

Fig. 6. Potential key frames from "star" video sequence extracted from each cluster of the shot.

TABLE I

VIDEO SET USED IN OUR EXPERIMENTS

\begin{tabular}{c|c|c|c|c}
\hline video & frames & cuts & fade-ins & fade-outs \\
\hline \hline basketball & 3882 & 44 & 7 & 4 \\
\hline news & 9446 & 40 & 6 & 6 \\
\hline football & 5589 & 28 & 0 & 0 \\
\hline movie & 19722 & 147 & 0 & 0 \\
\hline \hline \multicolumn{5}{c}{ TREC video sequences } \\
\hline 6 debate videos & 125977 & 230 & 0 & 0 \\
\hline 4 CNN news videos & 209978 & 1287 & 57 & 57 \\
\hline 4 ABC news videos & 206144 & 1269 & 64 & 69 \\
\hline
\end{tabular}

cluster $c_{2}$. Then we reassign the rest of key frames to these two clusters. If the MI of a key frame and the representative $k_{2}$ is higher than MI of this key frame and the representative $k_{1}$ of the cluster $c_{1}$ we assign this key frame to the cluster $c_{2}$. We repeat this procedure recursively, till we find all key frames.

\section{EXPERIMENTAL RESULTS AND DISCUSSION}

The proposed method was tested on several TV sequences (see Table I) containing many commercials, characterized by significant camera parameter changes like zoom-ins/outs, pans, abrupt camera movements as well as significant object and camera motion inside single shots. The video sequences contain film, sport, studio news, advertisements, political talks and TV series logos. These four video sequences "basketball," "news," "football," and "movie" totaled about $30 \mathrm{~min}$, their frame size varying between $176 \times 112$ and $176 \times 144$ pixels. These videos have a specific digitization feature: they frame content changes every third frame instead of every frame. For each video sequence, a human observer determined the precise locations and duration of the transitions to be used as ground truth (GT).

To enable future comparison with other boundary detection techniques, newscasts from the reference video test set TRECVID 2003 was added to the testing set, containing video sequences of more than $6 \mathrm{~h}$ duration that has been digitized with a frame rate of $29.97 \mathrm{fps}$ at a resolution of $352 \times 264$ pixels. We used spatially downsampled frames for our experiments with resolution $176 \times 132$ pixels to speed up calculations. The GT provided by TRECVID was used for these video sequences.

In order to evaluate the performance of the shot cut detection method presented in Section III, the following measures were used, inspired by the receiver operating characteristics in statistical detection theory [3], [32]. Let GT denote the ground truth, Det the detected (correct and false) shots cuts using our methods. The following performance measures have been used. 


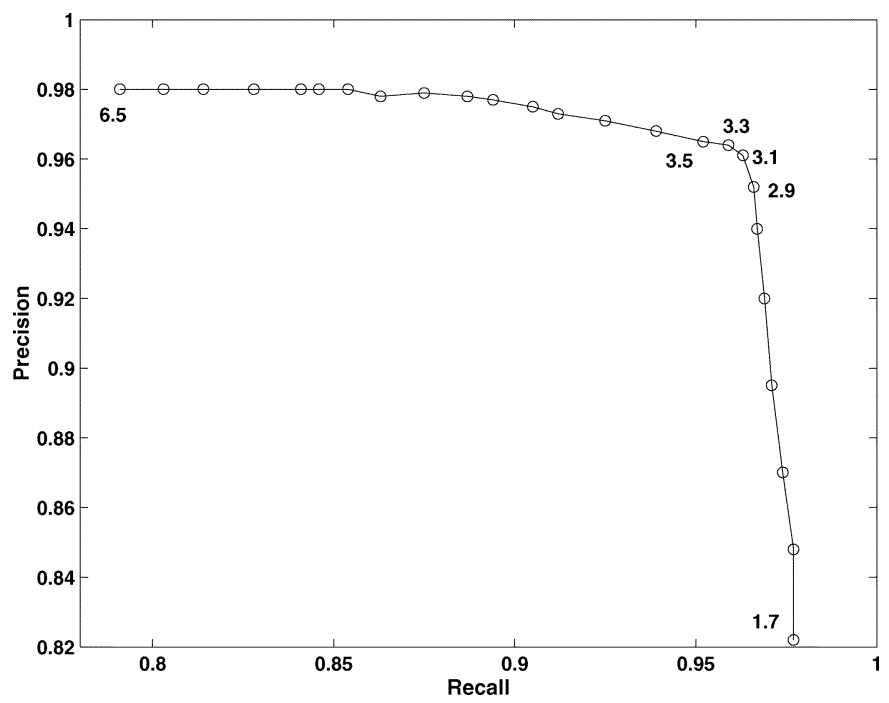

Fig. 7. Recall-precision graph obtained for shot cut detection method by varying threshold $\epsilon_{c}$ in the range [1.7, 6.5].

TABLE II

FiXed Threshold SHOT Cut Detection RESUlts

\begin{tabular}{|c|c|c|c|c|c|c|}
\hline & \multicolumn{2}{|c|}{ MI method } & \multicolumn{2}{|c|}{$\begin{array}{c}\text { Combined } \\
\text { histogram method }\end{array}$} & \multicolumn{2}{|c|}{$\begin{array}{c}\text { Color histogram } \\
\text { method }\end{array}$} \\
\hline video & Recall & Precision & Recall & Precision & Recall & Precisior \\
\hline basketball & 1.00 & 1.00 & 0.91 & 0.97 & 0.52 & 0.85 \\
\hline news & 0.96 & 1.00 & 0.96 & 98 & 0.80 & 0.89 \\
\hline football & 0.93 & 1.00 & 0.96 & 1.00 & 0.68 & 0.68 \\
\hline movie & 1.00 & 1.00 & 0.93 & 0.98 & 0.87 & 0.93 \\
\hline 6 debate vid & 1.00 & 0.99 & 1.00 & 1.00 & 0.87 & 1.00 \\
\hline $4 \mathrm{CNN}$ new & 0.96 & 96 & 0.87 & 0.83 & 0.85 & 0.84 \\
\hline 4 ABC news & 0.97 & 0.94 & 0.85 & 0.81 & 0.87 & 0.73 \\
\hline TREC total & 0.97 & 0.95 & "0.87 & 0.83 & 0.86 & 0.80 \\
\hline
\end{tabular}

- The Recall measure, also known as the true positive function or sensitivity, that corresponds to the ratio of correct experimental detections over the number of all true detections:

$$
\text { Recall }=\frac{|\operatorname{Det} \bigcap \mathrm{GT}|}{|\mathrm{GT}|}
$$

where $|\mathrm{GT}|$ denotes the cardinality of set GT.

- The Precision measure defined as the ratio of correct experimental detections over the number of all experimental detections

$$
\text { Precision }=\frac{|\operatorname{Det} \bigcap \mathrm{GT}|}{|\operatorname{Det}|}
$$

For our experiments we used the size of temporal window $N_{W}=3$. We have tested the method with several choices of threshold $\epsilon_{c}$. The recall-precision curve obtained by changing threshold $\epsilon_{c}$ is shown in Fig. 7. The experimental tests, performed using a common prefixed threshold $\left(\epsilon_{c}=3.1\right)$ for all video sequences are summarized in Table II (MI method). The elapsed time for obtaining results (abrupt cuts and fades) for one video sequence having 51384 frames was 1517 seconds. Thus, the algorithm can operate in real time for the raw video sequence. The large majority of the cuts were correctly detected even in the case of the video sequences, which contain fast object and camera movements. A part of the video sequence showing a cut between two shots involving high content motion

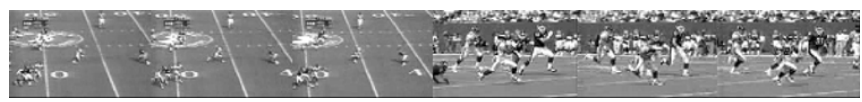

Fig. 8. Consecutive frames from "football" video sequence showing an abrupt cut between two shots coupled with large video object motion.

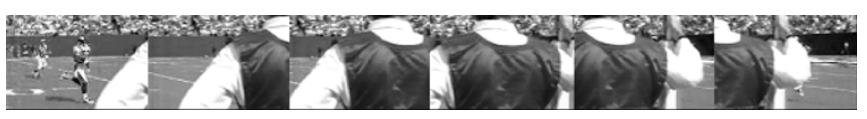

Fig. 9. Consecutive frames from "football" video sequence showing a big object appearance in front of the camera during panning.

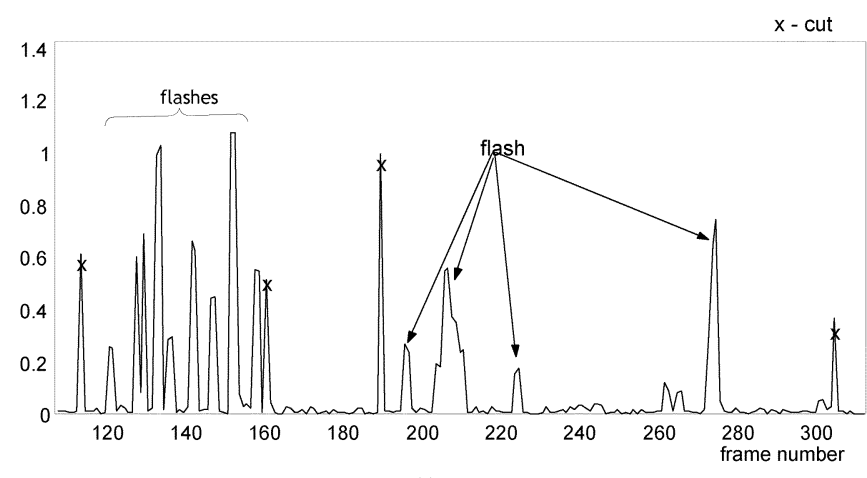

(a)

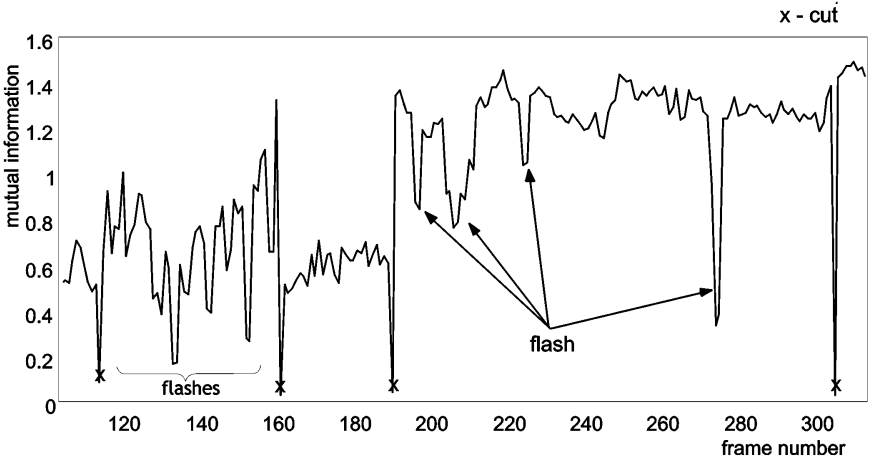

(b)

Fig. 10. Part of video sequence containing many camera flashes. (a) Color histogram comparison and (b) MI calculated for the same part of video sequence.

that was successfully detected by the proposed method is presented in Fig. 8. A snapshot of the "football" sequence is shown in Fig. 9, where a big object appears in front of the camera. This case is wrongly characterized by existing methods as a transition, whereas our method correctly does not detect a transition.

Sensitivity to camera flashes is shown on Fig. 10. Fig. 10(a)-(b) shows the color histogram difference and MI, respectively, calculated for the same part of video sequence containing a lot of camera flashes. Notice that the MI metric is significantly less sensitive to shot illumination changes (camera flashes) even in the RGB color space compared to histogram-based methods. This is clear from (6) and the properties of conditional entropy. In case of camera flash occurrence, the main information transported from one frame to the next one is preserved, which means that they differ only in luminance by a certain amount. Thus, from properties of conditional entropy $H(X \mid Y) \cong 0$ and accordingly $I(X, Y) \cong H(X)$. In the case of shot cuts the two frames are independent and there is no information transported between them, which means 


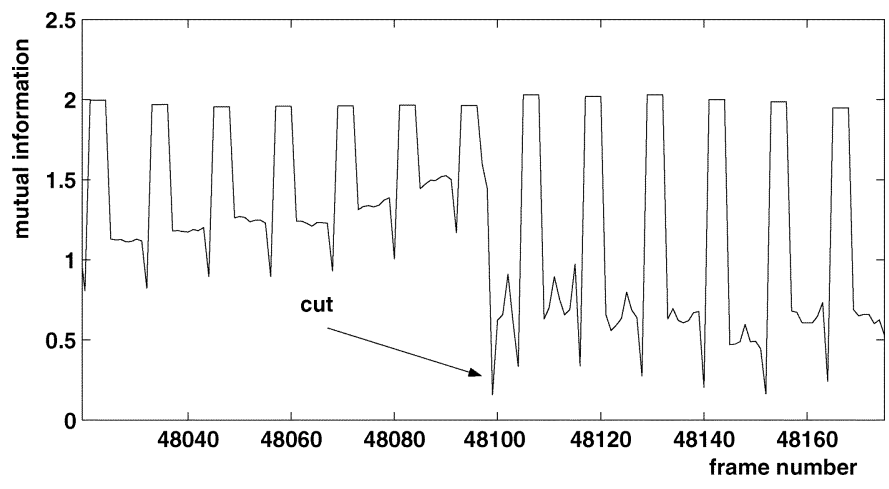

Fig. 11. MI of temporally subsampled video sequence.
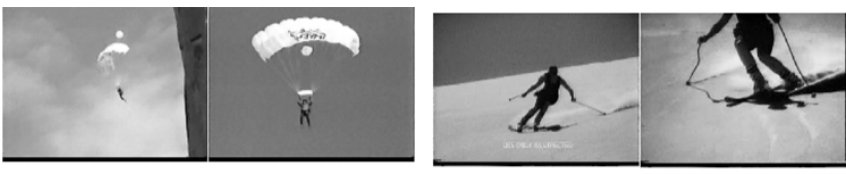

(a)
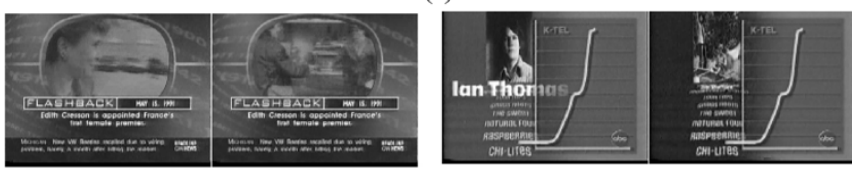

(b)

Fig. 12. Consecutive frames from video sequences presenting abrupt cuts, which caused missed shot cut detection. (a) Shot changes between two images with very similar spatial color distribution and (b) shot change occurs only in a part of the video frame.

$I(X, Y)=0$. In the case of histogram comparisons, the camera flashes sometimes produce more significant peaks than the peaks corresponding to cuts, which cause false detections.

The obtained results are better than the results for shot cut detections reported in the TRECVID2003 competition [33]. The best reported abrupt cut detection results for recall and precision are $93 \%$ and $95 \%$, respectively, whereas our method produces $97 \%$ recall and $95 \%$ precision. Most false detections were caused by corrupted parts of video sequences, where a sequence of exactly the same frames is followed by a significantly different frame. This case is shown in Fig. 11. In some cases, false detections appeared in the case of commercials where artistic camera edits were used. The missed shot cut detections were caused mainly by shot changes between two images with very similar spatial color distribution [Fig. 12(a)] or if the shot change occurred only in a part of the video frame [Fig. 12(b)]. Some video sequences contained a special type of hard cut, which contained one transitional frame ("cut in two frames"). In this case, the MI shows a double peak, which was not always a strong one. Therefore, in some cases, its use caused misdetection. Such fake peaks could be caused, for example, by flash. This drawback could be improved by a pre-processing stage, where all double MI peaks would be checked for the possibility of corresponding to a hard cut, by comparing the previous and the successive frame. In case the MI value is still small, we can modify it to become a single peak.

We compared our algorithm to the technique proposed in [22]. This approach combines two shot boundary detection schemes based on color frame differences and color vector histogram differences between successive frames. It is claimed to detect shot boundaries efficiently even under strong video edit effects and camera motion. This method operates in the HLS color space and ignores luminance information in order to overcome the possible drawback of histogram sensitivity to shot illumination changes. The results of this algorithm applied on the same video sequences are summarized in Table II (second and third columns). Several false shot cut detections were performed due to camera flashes. Although this approach has a high shot cut detection rate, it is generally lower compared to that of our proposed technique (Table II; MI method). Our technique is robust to the detection of shots with small length, occurring particularly during TV advertisements. Out of the falsely detected cuts, about $10 \%$ of them come from other types of transitions which were detected by chance.

Another algorithm, that was used for comparison purposes is the so-called color histogram-based shot boundary detection algorithm [2]. It is one of the most reliable variants of histogrambased detection algorithms. Hard cuts and other short-lasting transitions are detected as single peaks in the time series of the differences between color histograms of contiguous frames or of frames lying at a certain distance $k$ apart. A hard cut is detected if only the $i$ th color histogram difference value exceeds a certain threshold $\phi$ within a local environment of a given radius of frame $f_{i}$. A global thresholding is used. In order to cope with the particular type of hard cut, which consists of one transitional frame, double peaks were modified into single peaks in a pre-processing stage. Table II (fifth and sixth columns) shows the results obtained by this method with threshold $\phi=0.4$. One can see that, in general, the results are poorer than those of our proposed method using MI, they are even lower than the results obtained by the combined histogram method.

The same measures (17) and (18) have been used for the evaluation of fade detection performance. The spatial overlap precision has been used as well defined as

$$
\text { Spatial Overlap Precision }=\frac{1}{|\mathrm{GT} \cap \operatorname{Det}|} \sum_{i=1}^{|\mathrm{GT}|} \frac{\left|\operatorname{Det}_{i} \bigcap \mathrm{GT}_{i}\right|}{\left|\operatorname{Det}_{i} \bigcup \mathrm{GT}_{i}\right|}
$$

where $\mathrm{GT}_{i}$ is a subset of GT, which represents the duration of one fade. Overlap is considered to be a strong measure for detection accuracy, since, for example, a shot of length $N_{L}$ shifted by one frame results in only $\left(N_{L}-1\right) / N_{L}$ overlap.

The fade detection method was tested for several choices of threshold $\epsilon_{f}$. The recall-precision curve obtained by changing threshold $\epsilon_{f}$ is shown in Fig. 13. The experimental tests, performed using a common prefixed thresholds $\epsilon_{f}=0.15$ and $T=3$ ) for all video sequences are summarized in Table III. Using this setup, the fade boundaries were detected within a precision of \pm 2 frames. In most cases, the boundaries toward black frames were recognized without any error. The robustness of the JE measure in fade detection and, especially, in avoiding false fade detections is illustrated in Figs. 14 and 15. The use of JE for detecting fades is robust in case big objects move in front of the camera, that can cause severe occlusion and a blank frame in the video sequence. In our experiments, the threshold was set to a very low value to avoid false detections (see Fig. 15). Some fade detections were missed when there was noise in the black frame or when the fading was not complete and the end 


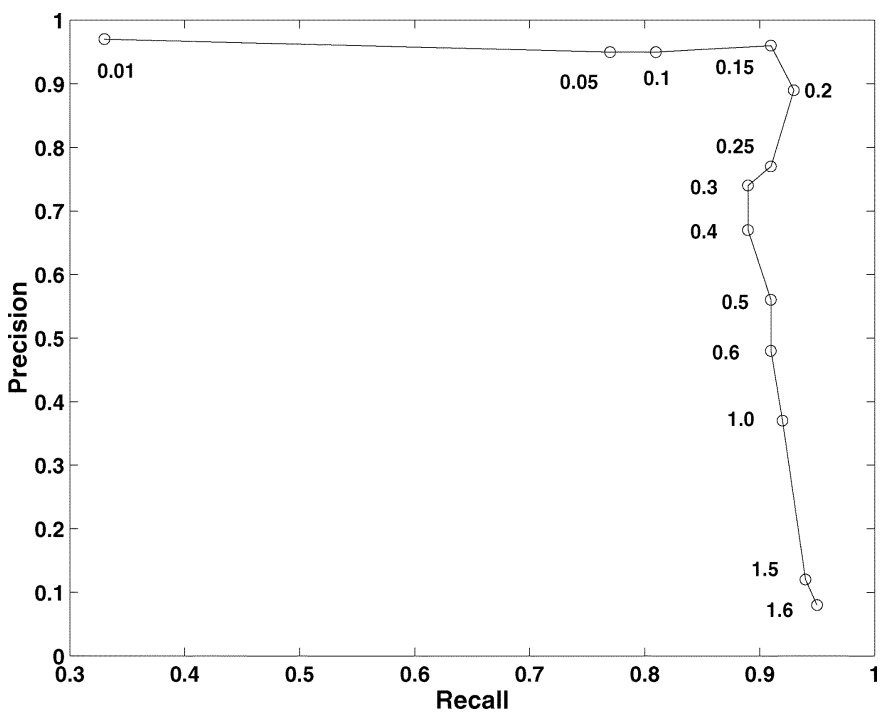

Fig. 13. Recall-precision graph obtained for fade detection method by varying threshold $\epsilon_{f}$ in the range $[0.01,1.5]$ and choosing $T=3$.

TABLE III

Evaluation of Fade Detection by the Proposed Joint Entropy Method

\begin{tabular}{c|c|c|c|c|c|c}
\hline \multirow{2}{*}{ video } & \multicolumn{3}{|c|}{ fade-ins } & \multicolumn{3}{c}{ fade-outs } \\
\cline { 2 - 7 } & Recall & Precision & Overlap & Recall & Precision & Overlap \\
\hline \hline basketball & $\mathbf{1 . 0 0}$ & 1.00 & $\mathbf{0 . 7 8}$ & $\mathbf{1 . 0 0}$ & 1.00 & $\mathbf{0 . 9 0}$ \\
\hline news & $\mathbf{1 . 0 0}$ & 1.00 & $\mathbf{0 . 7 1}$ & $\mathbf{1 . 0 0}$ & 1.00 & 0.85 \\
\hline \hline 4 CNN news & $\mathbf{1 . 0 0}$ & 0.84 & $\mathbf{0 . 8 3}$ & 1.00 & $\mathbf{0 . 8 4}$ & 0.86 \\
\hline 4 ABC news & 0.93 & $\mathbf{0 . 9 0}$ & $\mathbf{0 . 7 4}$ & 0.92 & $\mathbf{0 . 9 2}$ & 0.75 \\
\hline
\end{tabular}

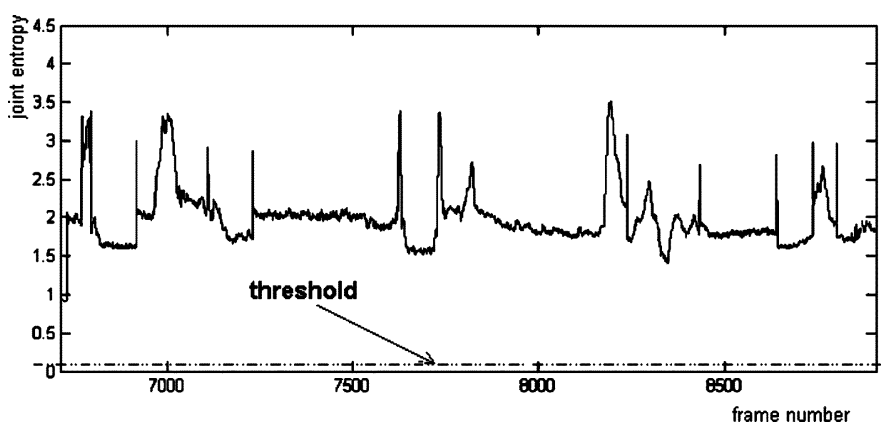

Fig. 14. JE signal from "star" video sequence representing no fades.

frame was just very dark gray instead of black. In the TRECVID competition, only the abrupt cuts and the gradual transitions are evaluated separately. Therefore, we cannot make comparison of our results to those of other TRECVID participants.

Our method for fade detection was compared to the approach proposed in [2] that is based on the standard deviation of pixel intensities (SD method) and claims to detect fades with high detection rate and to determine fade boundaries with high precision. In order to obtain results three different parameters have to be set: minimal required length of a fade, minimal required correlation and maximal allowed standard deviation of the pixel color values in the first/last monochrome frame of a fade. We used the default setup of the parameters in the algorithm, as proposed in [2], since it was referred that this choice attains the best performance. As can be seen in Table IV, several fades were not correctly detected by this method. You can notice a

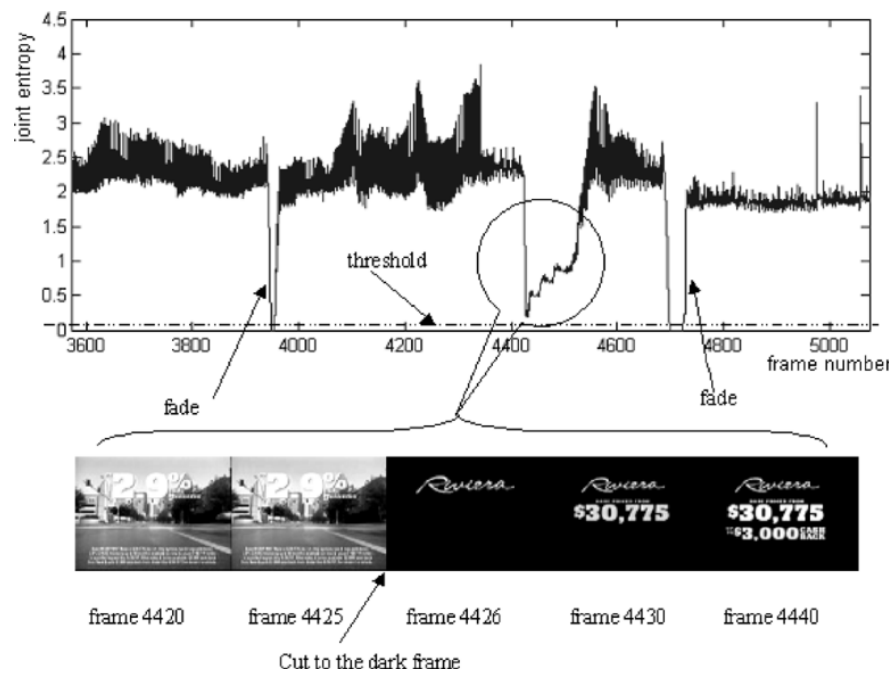

Fig. 15. JE signal from "news" video sequence having two fades.

TABLE IV

Evaluation of FAde DETECTION By THE SD METHOD [2]

\begin{tabular}{c|c|c|c|c|c|c}
\hline \multirow{2}{*}{ video } & \multicolumn{3}{|c|}{ fade-ins } & \multicolumn{3}{c}{ fade-outs } \\
\cline { 2 - 7 } & Recall & Precision & Overlap & Recall & Precision & Overlap \\
\hline \hline basketball & 0.14 & 1.00 & 0.41 & 0.50 & 1.00 & 0.85 \\
\hline news & 0.17 & 1.00 & 0.54 & 0.17 & 1.00 & 0.65 \\
\hline \hline 4 CNN news & 0.96 & 0.84 & 0.70 & 1.00 & 0.71 & 0.87 \\
\hline 4 ABC news & 1.00 & 0.88 & 0.68 & 0.97 & 0.87 & 0.81 \\
\hline
\end{tabular}

TABLE $\mathrm{V}$

FAde Location ERror Statistics For the Proposed JE Method (IN FRAME NUMBERS)

\begin{tabular}{c|c|c|c|c}
\hline effects & \multicolumn{2}{|c|}{ fade-outs } & \multicolumn{2}{c}{ fade-ins } \\
\hline frame & $f_{s}$ & $f_{e}$ & $f_{s}$ & $f_{e}$ \\
\hline median bias & 2 & $\mathbf{0}$ & $\mathbf{0}$ & 2 \\
\hline mean bias \pm s. dev. & $2.3 \pm 1.7$ & $\mathbf{0 . 2} \pm \mathbf{0 . 4}$ & $\mathbf{0 . 3} \pm \mathbf{0 . 6}$ & $\mathbf{2 . 6} \pm \mathbf{2 . 6}$ \\
\hline max bias & 9 & $\mathbf{1}$ & $\mathbf{2}$ & 10 \\
\hline
\end{tabular}

TABLE VI

FAde Location ERRor Statistics FOR the SD Method (IN FRAME NUMBERS)

\begin{tabular}{c|c|c|c|c}
\hline effects & \multicolumn{2}{|c|}{ fade-outs } & \multicolumn{2}{c}{ fade-ins } \\
\hline frame & $f_{s}$ & $f_{e}$ & $f_{s}$ & $f_{e}$ \\
\hline median bias & 1 & 1 & 1.5 & 1 \\
\hline mean bias \pm s. dev. & $1.7 \pm 2.3$ & $1.3 \pm 0.9$ & $1.8 \pm 2.2$ & $2.9 \pm 3.0$ \\
\hline max bias & 8 & 4 & 4 & 9 \\
\hline
\end{tabular}

very low recall in case of "basketball" and "news" video sequences, which was caused by the video content change every third frame. The above mentioned observations were also confirmed by the starting/ending frame location estimation provided by our JE (JE) approach and the SD technique and their error statistics are presented in Tables V and VI, respectively. For all fades (Table I), the starting and ending frames were detected by both methods and the location errors were calculated. The JE proposed method provided superior performance than the SD method in median and mean error values and presented no errors in fade-out end point detection. Furthermore, the significantly smaller maximum errors of the JE technique with regard to those of the SD method illustrate the robustness of our algorithm. 


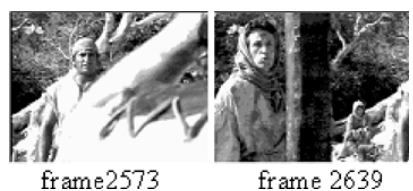

Fig. 16. Examples of key frames selected by our method from "star" video sequence to represent visual content of one shot.

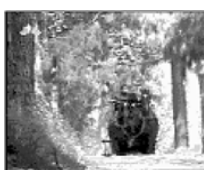

frame 314

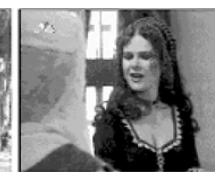

frame 2026

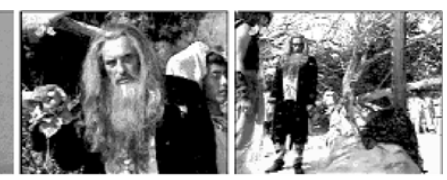

frame 2904 frame 4344
Fig. 17. Examples of key frames extracted by our method from "star" video sequence.

After the video was segmented to video shots, we applied our method for key frame selection on the video sequences. In the case of shots without significant content changes, our method successfully chose only one frame, even if more potential key frames were extracted after clustering. For shots with big content changes, usually due to camera or object motions, more key frames were selected, depending on visual complexity of the shot. An example of key frames extracted from one shot with more complicated content can be seen in Fig. 16. The selected key frames of a certain video sequence are shown in Fig. 17. Thus, our method captures the shot content very well.

\section{CONCLUSION}

A novel technique for shot transitions detection is presented. We propose a new method for detecting abrupt cuts and fades using the MI and the JE measures, respectively. The accuracy of our approach was experimentally shown to be very high. Experiments illustrated that fade detection using the JE can efficiently differentiate fades from cuts, pans, object, or camera motion and other types of video scene transitions, while most of the methods reported in the current literature fail to characterize these kinds of transitions. The method was successfully compared to other methods reported in literature.

\section{REFERENCES}

[1] X. U. Cabedo and S. K. Bhattacharjee, "Shot detection tools in digital video," in Proc. Non-Linear Model based Image Analysis, 1998, pp. 121-126.

[2] R. Lienhart, "Comparison of automatic shot boundary detection algorithms," in Proc. SPIE Storage and Retrieval for Image and Video Databases VII, vol. 3656, San Jose, CA, Jan. 1999, pp. 290-301.

[3] P. Browne, A. F. Smeaton, N. Murphy, N. O'Connor, S. Marlow, and C. Berrut, "Evaluation and combining digital video shot boundary detection algorithms," presented at the 4th Irish Machine Vision and Information Processing Conf., Belfast, Ireland, 2000.

[4] A. Dailianas, R. B. Allen, and P. England, "Comparison of automatic video segmentation algorithms," in Proc. SPIE Photonics East'95: Integration Issues in Large Commercial Media Delivery Systems, vol. 2615, Oct. 1995 , pp. 2-16.
[5] G. Ahanger and T. Little, "A survey of technologies for parsing and indexing digital video," J. Vis. Commun. Image Represent., vol. 7, no. 1, pp. 28-43, 1996.

[6] N. V. Patel and I. K. Sethi, "Video shot detection and characterization for video databases," Pattern Recognit., vol. 30, no. 4, pp. 583-592, Apr. 1997.

[7] S. Tsekeridou and I. Pitas, "Content-based video parsing and indexing based on audio-visual interaction," IEEE Trans. Circuits Syst. Video Technol., vol. 11, no. 4, pp. 522-535, Apr. 2001.

[8] C.-L. Huang and B.-Y. Liao, "A robust scene-change detection method for video segmentation," IEEE Trans. Circuits. Syst. Video Technol., vol. 11 , no. 12, pp. 1281-1288, Dec. 2001.

[9] A. Hanjalic, "Shot-boundary detection: Unraveled and resolved?," IEEE Trans. Circuits. Syst. Video Technol., vol. 12, no. 2, pp. 90-105, Feb. 2002.

[10] T. Butz and J. Thiran, "Shot boundary detection with mutual information," in Proc. 2001 IEEE Int. Conf. Image Processing, vol. 3, Oct. 2001, pp. 422-425.

[11] R. Lienhart, "Reliable dissolve detection," in Proc. SPIE Storage and Retrieval for Media Databases 2001, vol. 4315, Jan. 2001, pp. 219-230.

[12] M. S. Drew, Z.-N. Li, and X. Zhong, "Video dissolve and wipe detection via spatio-temporal images of chromatic histogram differences," in Proc. IEEE Int. Conf. Image Processing, vol. 3, 2000, pp. 929-932.

[13] R. Lienhart and A. Zaccarin, "A system for reliable dissolve detection in video," in Proc. IEEE Int. Conf. Image Processing 2001 (ICIP'01), Thessaloniki, Greece, Oct. 2001, pp. 406-409.

[14] R. Zabih, J. Miller, and K. Mai, "A feature-based algorithm for detecting and classifying production effects," ACM J. Multimedia Syst., vol. 7, pp. 119-128, 1999.

[15] Y. Wang, Z. Liu, and J.-C. Huang, "Multimedia content analysis using both audio and visual clues," IEEE Signal Process. Mag., vol. 17, no. 6, pp. 12-36, Nov. 2000.

[16] A. Hanjalic, "Shot-boundary detection: Unraveled and resolved?," IEEE Trans. Circuits Syst. Video Technol., vol. 12, no. 2, pp. 90-105, Feb. 2002.

[17] W. J. Heng and K. N. Ngan, "Shot boundary refinement for long transition in digital video sequence," IEEE Trans. Multimedia, vol. 4, no. 4, pp. 434-445, Dec. 2002.

[18] A. D. Bimbo, Visual Information Retrieval. San Mateo, CA: Morgan Kaufmann, 1999.

[19] A. Hampapur, R. Jain, and T. Weymouth, "Digital video segmentation," in Proc. ACM Multimedia, San Francisco, CA, Oct. 1994, pp. 357-364.

[20] A. M. Alattar, "Detecting fade regions in uncompressed video sequences," in Proc. IEEE Int. Conf. Acoustics, Speech, and Signal Processing, 1997, pp. 3025-3028.

[21] B. T. Truong, C. Dorai, and S. Venkatesh, "New enhancements to cut, fade, and dissolve detection processes in video segmentation," in ACM Multimedia 2000, Nov. 2000, pp. 219-227.

[22] S. Tsekeridou, S. Krinidis, and I. Pitas, "Scene change detection based on audio-visual analysis and interaction," in Proc. 2000 Multi-Image Search and Analysis Workshop, Mar. 2001, pp. 214-225.

[23] B. G. unsel and A. M. Tekalp, "Content-based video abstraction," in Proc. 1998 IEEE Int. Conf. Image Processing, Chicago, IL, Oct. 1998.

[24] A. Nagasaka and Y. Tanaka, "Automatic video indexing and full-video search for object appearances," Visual Database Syst., vol. II, 1992.

[25] W. Wolf, "Key frame selection by motion analysis," in Proc. 1996 IEEE Int. Conf. Acoust., Speech and Signal Proc., 1996, pp. 1228-1231.

[26] Y. Zhuang, Y. Rui, T. S. Huang, and S. Metrotra, "Adaptive key frame extraction using unsupervised clustering," in Proc. IEEE Int. Conf. Image Processing, Chicago, IL, Oct. 1998, pp. 886-890.

[27] T. M. Cover and J. A. Thomas, Elements of Information Theory. New York: Wiley, 1991.

[28] A. Papoulis, Probability, Random Variables, and Stochastic Processes. New York: McGraw-Hill, 1991.

[29] I. Pitas and A. Venetsanopoulos, Nonlinear Digital Filters: Principles and Applications. Norwell, MA: Kluwer, 1990.

[30] Z. Cernekova, C. Nikou, and I. Pitas, "Shot detection in video sequences using entropy-based metrics," in Proc. 2002 IEEE Int. Conf. Image Processing, Rochester, NY, Sep. 2002, pp. 421-424.

[31] C. Kotropoulos and I. Pitas, "A variant of learning vector quantizer based on split-merge statistical tests," in Proc. Lecture Notes in Computer Science:Computer Analysis of Images and Patterns. New York: Springer-Verlag, 1993.

[32] C. E. Metz, "Basic principles of ROC analysis," in Seminars in Nuclear Medicine, vol. 8, 1978, pp. 283-298.

[33] Trec Video Retrieval Evaluation (2003). [Online]. Available: http://www-nlpir.nist.gov/projects/trecvid/ 


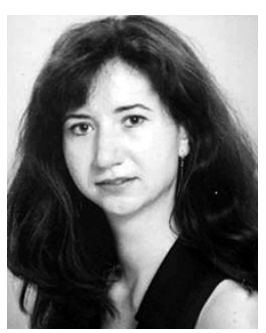

Zuzana Černeková received the M.S. degree and the Doctor of Natural Sciences (RNDr.) degree from Comenius University, Bratislava, Slovakia, in 1999 and 2000, respectively. She is currently a Researcher and working toward the Ph.D. degree at the Department of Informatics, Aristotle University of Thessaloniki, Thessaloniki, Greece.

She has studied informatics with specialization on mathematics methods of informatics and computer graphics. She was a Researcher and Lecture Assistant at the Department of Computer Graphics and Image Processing, Faculty of Mathematics and Physics, Comenius University. Her research interests lie in the areas of computer graphics, visualization, 3-D animations, multimedia, video processing and pattern recognition.

Ms. Cernekova is a member of the SCCG organizing committee.

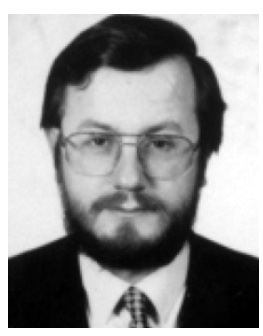

Ioannis Pitas (SM'94) received the Diploma of electrical engineering in 1980 and the Ph.D. degree in electrical engineering in 1985, both from the University of Thessaloniki, Thessaloniki, Greece.

Since 1994, he has been a Professor with the Department of Informatics, University of Thessaloniki. From 1980 to 1993, he served as Scientific Assistant, Lecturer, Assistant Professor, and Associate Professor with the Department of Electrical and Computer Engineering at the University of Thessaloniki. He served as a Visiting Research Associate at the University of Toronto, Toronto, ON, Canada; University of Erlangen-Nuernberg, Nuernberg, Germany; Tampere University of Technology, Tampere, Finland; as Visiting Assistant Professor at the University of Toronto; and as Visiting Professor at the University of British Columbia, Vancouver, BC, Canada. He was lecturer in short courses for continuing education. His current interests are in the areas of digital image processing, multidimensional signal processing, watermarking, and computer vision. He has published over 380 papers and contributed to 13 books in his areas of interest. He is the co-author of the books Nonlinear Digital Filters: Principles and Applications (Boston, MA: Kluwer, 1990), 3-D Image Processing Algorithms (New York: Wiley, 2000), Nonlinear Model-Based Image/Video Processing and Analysis (New York: Wiley, 2001), and author of Digital Image Processing Algorithms and Applications (New York: Wiley, 2000). He is the editor of the book Parallel Algorithms and Architectures for Digital Image Processing, Computer Vision and Neural Networks (New York: Wiley, 1993). He is co-editor of the journal Multidimensional Systems and Signal Processing

Dr. Pitas has been member of the European Community ESPRIT Parallel Action Committee. He has also been an invited speaker and/or member of the program committee of several scientific conferences and workshops. He was Associate Editor of the IEEE TRANSACTIONS ON CIRCUITS AND SYSTEMS, Associate Editor of the IEEE TRANSACTIONS ON NEURAL NETWORKS, and he is currently an Associate Editor of the IEEE TRANSACTIONS ON IMAGE PROCESSING. He was general chair of the 1995 IEEE Workshop on Nonlinear Signal and Image Processing (NSIP95), technical chair of the 1998 European Signal Processing Conference, and general chair of IEEE ICIP2001.

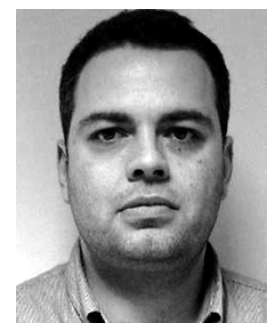

Christophoros Nikou (M'02) was born in Thessaloniki, Greece, in 1971. He received the Ph.D. degree in image processing and computer vision and the DEA degree in optoelectronics and image processing from Louis Pasteur University, Strasbourg, France, in 1995 and 1999, respectively, and the Diploma degree in electrical engineering from the Aristotle University of Thessaloniki, Thessaloniki, Greece, in 1994.

During 2001, he was a Senior Researcher with the Department of Informatics, Aristotle University of Thessaloniki, where he conducted research in image processing in the framework of various European projects. From 2002 to 2004, he was with Compucon S.A., Thessaloniki, Greece, managing research projects in 3-D medical image processing and analysis. Since 2004, he has been a Lecturer at the Department of Computer Science, University of Ioannina, Ioannina, Greece. His research interests mainly include computer vision, pattern recognition, biomedical image processing, image registration and segmentation, deformable models, and statistical image processing.

Dr. Nikou is a member of the Technical Chamber of Greece. 